\title{
UNDERSTANDING VIRAL DSRNA PRODUCTION, CELL ENTRY AND USE AS AN ANTIVIRAL THERAPEUTIC IN RAINBOW TROUT
}

\author{
Sarah Poynter ${ }^{1,2}$, Katelin Spiteri ${ }^{2}$, Amal Aloufi ${ }^{2}$, Jeremy Weleff ${ }^{2}$, Adam Soares ${ }^{2}$, Stephanie \\ DeWitte-Orr ${ }^{2} \S$ \\ ${ }^{1}$ Department of Biology, University of Waterloo, Waterloo, Ontario, Canada, N2L 3G1 \\ 2 Department of Health Sciences, Wilfrid Laurier University, Waterloo, Ontario, Canada, N2L \\ $3 C 5$
}

\begin{abstract}
It is commonly believed that all viruses produce double-stranded (ds)RNA during their replicative cycle, either as a genomic fragment, replicative intermediate or product of over-lapping transcription. While dsRNA production has been well characterized in human viral pathogens, very little is known of this molecule with regards to fish viruses. We have found that fish viruses make dsRNA during replication, including those with dsDNA genome (frog virus 3), ssRNA genome (VHSV-IVb) and dsRNA genome (chum salmon reovirus). These dsRNA molecules are made at times and in locations where the virus is replicating. Cell lysis occurs during many virus infections, at which time viral dsRNA would be released into the extracellular space. Class A scavenger receptors (SR-As), act as surface receptors for dsRNA in mammals. We have found that SR-As are present and function on rainbow trout cells derived from the gill (RTgill-W1) and gut (RTgutGC). Interesting, dsRNA entry is SR-A mediated in RTgutGC but not RTgill-W1; and while both cell lines respond to dsRNA and mount a type I interferon (IFN) response, RTgutGC's SR-A-dependent response occurs faster and stronger. Our goal is to harness the immunomodulatory potential of dsRNA to design better antiviral therapies and vaccine adjuvants. To this end, we have identified a carrier for dsRNA that enhances dsRNA-mediated responses, and triggers stronger IFN and interferon-stimulated gene (ISG) expression compared to dsRNA alone.
\end{abstract}

\section{KEYWORDS}

Rainbow trout, innate immunity, dsRNA, SR-As, type I interferons

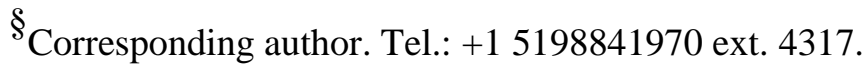

E-mail address: sdewitteorr@wlu.ca 